\title{
屠规彰方程组的 $\boldsymbol{N}$ 孤立子解
}

屠规彰 (Phys. Lett., 94A (1983), 340-342) 构造了如下方程组

$$
\left\{\begin{array}{l}
U_{l}=U_{x}+2 V, \\
V_{l}=-2 U V,
\end{array}\right.
$$

我们求得了这个方程组的 $N$ 孤立子解为

$$
\left\{\begin{array}{l}
U=K\left(\operatorname{Tr}\left(\frac{2 B}{1+B}\right)-1\right), \\
V=2 K^{2} \operatorname{Tr}\left(\frac{B A}{(1+B)^{2}}\right),
\end{array}\right.
$$

其中 $A$ 与 $B$ 均为 $N \times N$ 矩阵, $B_{i j}=\phi_{i} \phi_{j}$.

$$
A=\operatorname{diag}\left[1-\frac{d}{d x} \alpha_{1}(x), 1-\frac{d}{d x} \alpha_{2}(x),\right.
$$

$\left.\cdots, 1-\frac{d}{d x} \alpha_{N}(x)\right], \quad \phi_{i}=\exp \left[K\left(t+\alpha_{i}(x)\right)\right]$,

$T r$ 是矩阵的迹, $K$ 为任意实数 $r, \alpha_{i}(x)$ 为不恒等于 $x$ 的任意函数.

李竘神、田畴(科学通报,29(1984),318)曾得到 单私立子解为

$$
\left\{\begin{array}{l}
U=K \operatorname{th}\{K[t+\alpha(x)]\}, \\
V=\frac{1}{2} K^{2}\left(1-\frac{d \alpha(x)}{d x}\right) \operatorname{sech}^{2}\{K[t+\alpha(x)]\},
\end{array}\right.
$$

此与我们求得结果中 $N=1$ 的情况相一致.

郑维民

（上海计算技术研究所）

\section{中立型微分方程解的振动性}

我们考虑几类中立型线性微分方程

$$
\begin{gathered}
x^{\prime}(t)+\sum_{i=1}^{m} p_{i} x^{\prime}\left(t-\tau_{i}\right)+q x(t-\sigma)=0, \text { (1) } \\
x^{\prime}(t)+\delta p x^{\prime}(t-\tau)-\sum_{i=1}^{m} \delta q_{i} x\left(t+\delta \tau_{i}\right)=0,(2) \\
x^{\prime}(t)-p x^{\prime}(t-\tau)+q x(t-\tau) \\
+r x(t+\tau)=0,
\end{gathered}
$$

其中 $t \geqslant t_{0}$, 解的振动性质, 现把结果摘要如下:

定理 1 假设 $p_{i}, q, \tau_{i}$ 和 $\sigma$ 都是正数且 $\sigma>$ $z_{i}, i=1,2, \cdots, m$, 还设

$$
e q>\frac{1}{\sigma}+\sum_{i=1}^{m} \frac{p_{i}}{\sigma-\tau_{i}},
$$

则(1)式的一切解振动 (均指右向振动).

定理 2 假设 $p, q_{i}, \tau$ 和 $\tau_{i}$ 都是正数, 且 $\delta= \pm 1$ 和

$$
\frac{\sum_{i=1}^{m} q_{i}\left[1+\ln \left(\sum_{i=1}^{m} q_{i} \tau_{i}\right)\right]}{\sum_{i=1}^{m} q_{i} \tau_{i}}>\frac{p}{\tau e},
$$

则(2)式的一切解振动.

定理 3 假设 $p, q, r$ 和 $\tau$ 都是正数, 又设若 存在 $N_{1}>0, N_{2}<0$ 且 $N_{1}+N_{2}=1$ 使 $\frac{N_{1}}{\tau}\left[1-\ln \frac{N_{1}}{q \tau}\right]+\frac{N_{2}}{\tau}\left[\ln \left(\frac{-N_{2}}{\tau r}\right)-1\right]>\frac{p}{\tau e}$,
则(3)的一切解振动.

推论; 若满足

$$
\frac{1}{\tau}+\tau r-\left(r+\frac{1}{\tau}\right) \ln \frac{1+\tau r}{q \tau}>\frac{p}{\tau e}
$$

或者满足

$$
\frac{1+\tau}{\tau}\left[1-\ln \frac{1+\tau}{q \tau}\right]+(\ln r+1)>\frac{p}{\tau \epsilon},
$$

则(3)的一切解振动.

注 1 在情况 $m=1$, 条件 (4) 改进了最近 Grammatikopouls 等人得到的结果.

注 2 在 $p_{i}=0, i=1 、 2, \cdots, m$ 时,定理 1 和 定理 2 都与已知的有关时滞方程的结果一致.

注 3 定理 2 适用于情况 $\tau=\tau_{i}, i=1$, 在某 些情况下比阮昫(科学通报，1985, N.3) 的结果要 好.

这些定理的证明都是用一个统一的方法, 即寻 找充分条件，使其特征方程(一类超越方程)没有实 根. 本文所采用的方法在处理多个偏差变元时特别 有效.

\section{毛士忠}

（盐城师专数学系, 江苏)

张炳根 管征源

(山东海洋学院数学系,青岛) 